\title{
LOGARITHMS AND EXPONENTIALS IN BANACH ALGEBRAS
}

\author{
RAYMOND MORTINI ${ }^{1 *}$ AND RUDOLF RUPP ${ }^{2}$
}

\begin{abstract}
Let $A$ be a complex Banach algebra. If the spectrum of an invertible element $a \in A$ does not separate the plane, then $a$ admits a logarithm. We present two elementary proofs of this classical result which are independent of the holomorphic functional calculus. We also discuss the case of real Banach algebras. As applications, we obtain simple proofs that every invertible matrix over $\mathbb{C}$ has a logarithm and that every real matrix $M$ in $M_{n}(\mathbb{R})$ with $\operatorname{det} M>0$ is a product of two real exponential matrices.
\end{abstract}

12.7.2018

\section{Complex Banach algebras}

The purpose of this note is to show that the following important result in the realm of Banach algebras can also be proven by elementary methods which are independent of the holomorphic functional calculus. For the classical proof, see for instance [4, Theorem 10.30, p. 264].

Theorem 1.1. Let $A$ be a unital complex Banach algebra (not necessarily commutative) and let $a \in A$. Suppose that 0 belongs to the unbounded connected component of $\mathbb{C} \backslash \sigma_{A}(a)$, where $\sigma_{A}(a)$ is the spectrum of a. Then there exists $b \in A$ such that $e^{b}=a$.

We shall only use the following standard result which can be found in almost all monographs on functional analysis and Banach algebras.

Lemma 1.2. Let $A=(A,\|\cdot\|)$ be a unital, real or complex, but not necessarily commutative Banach algebra, the norm $\|\cdot\|$ being submultiplicative and let

$$
\exp A=\left\{e^{a}: a \in A\right\} .
$$

Suppose that $f \in A$ satisfies $\|\mathbf{1}-f\|<1$. Then $f \in \exp A$. Moreover, if $L$ is given by

$$
L=-\sum_{j=1}^{\infty} \frac{1}{j}(1-f)^{j},
$$

Date: Received: xxxxxx; Revised: yyyyyy; Accepted: zzzzzz.

* Corresponding author.

2010 Mathematics Subject Classification. Primary 46H05, Secondary 46J05, 15A16.

Key words and phrases. Real and complex Banach algebras; logarithms; exponentials; matrices. 
then the series is unconditionnally/absolutely convergent in $A$ and $e^{L}=f$. Finally, if for some $g \in A, M>\|g\|$, then

$$
M \cdot \mathbf{1}-g \in \exp A^{1},
$$

and in the case of a complex algebra,

$$
g-\lambda \cdot \mathbf{1} \in \exp A
$$

for every $\lambda \in \mathbb{C}$ with $|\lambda|>\|g\|$.

Proof. For the reader's convenience, we prove the latter assertion. Observe that

$$
h:=M \cdot \mathbf{1}-g=M(\mathbf{1}-(g / M)) .
$$

Now apply the first assertion to $f:=\mathbf{1}-(g / M)$. Then $f=e^{a}$ for some $a \in A$. Let $m:=\log M$ be a real $\log a r i t h m$ of $M$. Since $m \cdot \mathbf{1}$ commutes with each $a \in A$, we deduce that

$$
h=e^{m \cdot 1} e^{a}=e^{m \cdot 1+a} \in \exp A .
$$

If $\mathbb{K}=\mathbb{C}$, we let $m$ be a fixed complex logarithm of $\lambda$. Then

$$
g-\lambda \cdot \mathbf{1}=-(\lambda \cdot \mathbf{1}-g)=-e^{m \cdot \mathbf{1}+a}=e^{(i \pi+m) \cdot \mathbf{1}+a} \in \exp A .
$$

\section{First proof of Theorem 1.1.}

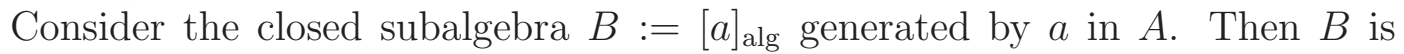
a commutative complex Banach algebra. Note that $\sigma_{B}(a)$ is contained in the polynomial convex hull of $\sigma_{A}(a)$ (see [4, Theorem 10.18 (b), p. 256]) ${ }^{2}$. In particular, 0 stays in the unbounded component of $\mathbb{C} \backslash \sigma_{B}(a)$. Hence, there is an arc $J$ in $\mathbb{C} \backslash \sigma_{B}(a)$ given by the parametrization $\varphi:\left[0,1\left[\rightarrow \mathbb{C} \backslash \sigma_{B}(a)\right.\right.$ joining 0 to infinity. Since $\Phi(t):=a-\varphi(t) \cdot \mathbf{1} \in B^{-1}$ for every $t \in[0,1[$, and $\Phi(t) \in \exp B$ for all $t$ sufficiently close to 1 (Lemma 1.2), the fact that $\exp B$ is a maximal connected subset of $B^{-1}$ now implies that $\Phi(t) \in \exp B$ for every $t$. In particular, $\Phi(0)=a \in \exp B$. Because $B \subseteq A$, we have found $b \in A$ such that $e^{b}=a$.

Yet an even more elementary proof can be given along the following lines. It avoids the necessity to know that the complement of the spectrum of $a$ with respect to the algebra $B=[a]_{\text {alg }}$ has the same unbounded component as the complement of the spectrum of $a$ with respect to the 'big' algebra $A$. This second proof only makes use of Lemma 1.2 and a simple connectedness argument. It is suitable for an undergraduate course in analysis and linear algebra.

\section{Second proof of Theorem 1.1.}

Let $J^{\prime}$ be an arc in $\mathbb{C} \backslash \sigma_{A}(a)$ joining 0 to infinity and which is given by the parametrization $\varphi:\left[0,1\left[\rightarrow \mathbb{C} \backslash \sigma_{A}(a)\right.\right.$. Let us emphasize that in contrast to the first proof, the arc lives in $\mathbb{C} \backslash \sigma_{A}(a)$. As above, let $B:=[a]_{\text {alg }}$ be the closed subalgebra of $A$ generated by $a$. Associate with $\varphi$ the path $\Phi:\left[0,1\left[\rightarrow A^{-1} \cap B\right.\right.$ given by

$$
\Phi(t):=a-\varphi(t) \cdot \mathbf{1}
$$

\footnotetext{
${ }^{1}$ Note that in the real case we do not necessarily have that the opposite $g-M \cdot \mathbf{1}$ belongs to $\exp A$.

${ }^{2}$ We actually have that $\sigma_{B}(a)=\widehat{\sigma_{A}(a)}$.
} 
and consider the set

$$
\mathcal{O}=\{t \in[0,1[: \Phi(t) \in \exp B\} .
$$

Since by Lemma 1.2, $\Phi(t) \in \exp B$ for $t$ close to $1, \mathcal{O} \neq \emptyset$.

Claim $1 \mathcal{O}$ is open in $[0,1[$.

To see this, let $t_{1} \in \mathcal{O}$. Then $\Phi\left(t_{1}\right)=e^{h}$ for some $h \in B$. Let $0<\varepsilon<\left\|e^{-h}\right\|^{-1}$ and choose a neighborhood $I_{\delta} \subseteq\left[0,1\left[\right.\right.$ of $t_{1}$ such that

$$
\left|\varphi(t)-\varphi\left(t_{1}\right)\right|<\varepsilon \text { for } t \in I_{\delta} \text {. }
$$

Then, for these $t$,

$$
\left\|\Phi(t)-\Phi\left(t_{1}\right)\right\|=\left\|\varphi(t) \cdot \mathbf{1}-\varphi\left(t_{1}\right) \cdot \mathbf{1}\right\|=\left|\varphi(t)-\varphi\left(t_{1}\right)\right|<\varepsilon .
$$

Hence

$$
\left\|e^{-h} \Phi(t)-\mathbf{1}\right\|=\left\|e^{-h}\left(\Phi(t)-e^{h}\right)\right\| \leq\left\|e^{-h}\right\| \varepsilon<1 .
$$

By Lemma 1.2, $e^{-h} \Phi(t)=e^{g}$ for some $g \in B$; choose for example

$$
g=\sum_{n=1}^{\infty}(-1)^{n+1} \frac{1}{n}\left(e^{-h} \Phi(t)-\mathbf{1}\right)^{n} .
$$

Since $B$ is commutative, $\Phi(t)=e^{h} e^{g}=e^{h+g} \in \exp B$. Thus $I_{\delta} \subseteq \mathcal{O}$.

Claim $2 \mathcal{O}$ is closed in $[0,1[$.

Let $t_{n} \in \mathcal{O}$ converge to some $s \in\left[0,1\right.$. By definition of $\mathcal{O}, \Phi\left(t_{n}\right) \in \exp B$; say $\Phi\left(t_{n}\right)=e^{h_{n}}$ with $h_{n} \in B$. As in the previous paragraph,

$$
\left\|\Phi\left(t_{n}\right)-\Phi(s)\right\| \leq\left|\varphi\left(t_{n}\right)-\varphi(s)\right| \rightarrow 0 .
$$

Since inversion is a continuous operation in $A^{-1}$ and $\Phi\left(\left[0,1[) \subseteq A^{-1}\right.\right.$, we conclude from $\left\|\Phi\left(t_{n}\right)-\Phi(s)\right\| \rightarrow 0$, that $\left\|\Phi\left(t_{n}\right)^{-1}-\Phi(s)^{-1}\right\| \rightarrow 0$. In particular, for all $n$,

$$
\left\|e^{-h_{n}}\right\|=\left\|\Phi\left(t_{n}\right)^{-1}\right\| \leq M<\infty \text {. }
$$

Now let $0<\varepsilon<1 / M$. Choose $n_{0}$ so that $\left|\varphi\left(t_{n}\right)-\varphi(s)\right|<\varepsilon$ for $n \geq n_{0}$. Then for all $n \geq n_{0}$,

$$
\begin{gathered}
\left\|e^{-h_{n}} \Phi(s)-\mathbf{1}\right\|=\left\|e^{-h_{n}}\left(\Phi(s)-e^{h_{n}}\right)\right\| \leq M\left\|\Phi(s)-e^{h_{n}}\right\| \\
\leq M\left|\varphi(s)-\varphi\left(t_{n}\right)\right| \leq M \varepsilon<1 .
\end{gathered}
$$

By Lemma $1.2 e^{-h_{n_{0}}} \Phi(s)=e^{k}$ for some $k \in B$; choose for example

$$
k=\sum_{n=1}^{\infty}(-1)^{n+1} \frac{1}{n}\left(e^{-h_{n}} \Phi(s)-\mathbf{1}\right)^{n} .
$$

Since $B$ is commutative,

$$
\Phi(s)=e^{h_{n_{0}}} e^{k}=e^{h_{n_{0}}+k} .
$$

Thus $s \in \mathcal{O}$ and so $\mathcal{O}$ is closed. Due to connectedness, we deduce that $\mathcal{O}=[0,1[$. Hence $a=\Phi(0) \in \exp B$.

Thus, we also obtain an easy proof (without the functional calculus or the use of the Jordan decomposition) of the following well-known result: 
Corollary 1.3. Let $M_{n}(\mathbb{C})$ be the algebra of $n \times n$-matrices over $\mathbb{C}$. Then each invertible matrix $M$ is an exponential matrix.

Proof. Since the spectrum of $M$ is finite, we see that the hypotheses of the previous theorem are all satisfied.

\section{REAL BANACH ALGEBRAS}

Let $\mathcal{R}$ be a real Banach algebra with unit element 1 . If $x \in \mathcal{R}$, then its (real-symmetric) spectrum $\sigma_{\mathcal{R}}^{*}(x)$ is defined as

$$
\begin{aligned}
\sigma_{\mathcal{R}}^{*}(x): & =\{\lambda \in \mathbb{C}:(a-\lambda \cdot \mathbf{1})(a-\bar{\lambda} \cdot \mathbf{1}) \text { not invertible in } \mathcal{R}\} \\
& =\left\{\lambda \in \mathbb{C}: a^{2}-2(\operatorname{Re} \lambda) a+|\lambda|^{2} \cdot \mathbf{1} \text { not invertible in } \mathcal{R}\right\} .
\end{aligned}
$$

Note that $\sigma_{\mathcal{R}}^{*}(x)$ is a non-void compact set in $\mathbb{C}$ (see for example [3]). This definition, going back to Kaplansky, can be motivated by looking at the characteristic polynomial of a real matrix $M$. Here the zeros of

$$
p_{n}(\lambda):=\operatorname{det}\left(M-\lambda I_{n}\right)
$$

are either real or appear in pairs $(\lambda, \bar{\lambda})$. Hence $\operatorname{det}\left(M-\bar{\lambda} I_{n}\right)$ has the same zeros. Since $M-\lambda I_{n}$ is not a real matrix, one considers $N:=\left(M-\lambda I_{n}\right)\left(M-\bar{\lambda} I_{n}\right)$, which has the property that $\operatorname{det} N=p_{n}(\lambda)^{2}$. What we have gained is that $N=M^{2}-2(\operatorname{Re} \lambda) M+|\lambda|^{2} I_{n}$ is a real matrix again. Here is now the companion result to Theorem 1.1.

Theorem 2.1. Let $\mathcal{R}$ be a unital real Banach algebra (not necessarily commutative) and let $x \in \mathcal{R}$.

(1) Suppose that 0 belongs to the unbounded connected component of $\mathbb{C} \backslash \sigma_{\mathcal{R}}^{*}(x)$. Then there exists $r \in \mathcal{R}$ such that $e^{r}=x^{2}$.

(2) If $]-\infty, 0]$ belongs the unbounded connected component of $\mathbb{C} \backslash \sigma_{\mathcal{R}}^{*}(x)$, then there exists $v \in \mathcal{R}$ such that $e^{v}=x$.

Proof. (1) We first note that the element $u:=x^{2}-2(\operatorname{Re} M) x+|M|^{2} \cdot \mathbf{1}$ is an exponential in $\mathcal{R}$ whenever $M \in \mathbb{C}$ is large enough. In fact, since

$$
f:=\frac{u}{|M|^{2}}=\left(\frac{x}{|M|}\right)^{2}-2\left(\operatorname{Re} \frac{M}{|M|}\right) \frac{x}{|M|}+\mathbf{1} \in \mathcal{R}
$$

and satisfies $\|f-\mathbf{1}\|<1$ for $M$ large enough, we deduce from Lemma 1.2 that $f=e^{L}$ for some $L \in \mathcal{R}$. Hence $u=e^{2 \log |M| \cdot 1+L}$ is the desired representation of $u$ (again because $2 \log |M| \cdot \mathbf{1}$ and $L$ commute).

Now the proof works in exactly the same way as before; just associate to the $\operatorname{arc} \varphi:\left[0, \infty\left[\rightarrow \mathbb{C} \backslash \sigma_{\mathcal{R}}^{*}(x)\right.\right.$ which joins 0 to $\infty$, the path $\Phi:\left[0, \infty\left[\rightarrow \mathcal{R}^{-1} \cap[x]_{\mathrm{alg}}\right.\right.$ given by

$$
\Phi(t):=(x-\varphi(t) \cdot \mathbf{1})(x-\overline{\varphi(t)} \cdot \mathbf{1})=x^{2}-2(\operatorname{Re} \varphi(t)) x+|\varphi(t)|^{2} \cdot \mathbf{1} .
$$

(2) By assumption, $\Phi(t)=x+t \cdot \mathbf{1} \in \mathcal{R}^{-1}$ for every $t \in[0, \infty[$. Moreover, for $M>0$ large,

$$
\Phi(M)=x+M \cdot \mathbf{1}=M\left(\frac{x}{M}+\mathbf{1}\right)
$$


has a logarithm by Lemma 1.2 applied to $f:=\frac{x}{M}+\mathbf{1}$. The conclusion now follows using the previous arguments.

Corollary 2.2. Let $\mathcal{R}$ be a unital real Banach algebra (not necessarily commutative) and let $x \in \mathcal{R}$. Suppose that 0 belongs to the unbounded connected component of $\mathbb{C} \backslash \sigma_{\mathcal{R}}^{*}(x)$. Then

$$
x \in \exp \mathcal{R} \text { if and only if } x=y^{2} \text { for some } y \in \mathcal{R} .
$$

Proof. If $x=e^{b}$ for some $b \in \mathcal{R}$, then we choose $y=e^{b / 2}$. Conversely, if $x=y^{2}$, then, by the spectral mapping theorem in its most primitive version,

$$
\sigma_{\mathcal{R}}^{*}(x)=\sigma_{\mathcal{R}}^{*}\left(y^{2}\right)=q\left(\sigma_{\mathcal{R}}^{*}(y)\right),
$$

where $q(z)=z^{2}$ (see for example [3]). Let $\varphi:\left[0, \infty\left[\rightarrow \mathbb{C} \backslash \sigma_{\mathcal{R}}^{*}(x)\right.\right.$ be a polygonial Jordan arc joining 0 with $\infty$. We may use polar-coordinates: so $\varphi(t)=r(t) e^{i s(t)}$ for some continuous functions $r$ and $s, r>0$ on $] 0, \infty[$ and $r(0)=0$. Then the arc $\psi:\left[0, \infty\left[\rightarrow \mathbb{C}\right.\right.$ given by $\psi(t):=\sqrt{r(t)} e^{i s(t) / 2}$ does not meet $\sigma_{\mathcal{R}}^{*}(y)$, because otherwise $q\left(\psi\left(t_{0}\right)\right)=\varphi\left(t_{0}\right) \in \sigma_{\mathcal{R}}^{*}(x)$. Hence, by Theorem $2.1(1), x=y^{2} \in$ $\exp \mathcal{R}$.

Let us emphasize that $x$ (or $-x$ ) itself does not necessarily have a real logarithm. Just look at the algebra $M_{3}(\mathbb{R})$ of $3 \times 3$ matrices over $\mathbb{R}$ and the diagonal matrix

$$
M=\left(\begin{array}{ccc}
-1 & 0 & 0 \\
0 & -2 & 0 \\
0 & 0 & 1
\end{array}\right) .
$$

Note that $\operatorname{det} M>0$. Suppose that there is $L \in M_{3}(\mathbb{R})$ such that $M=e^{L}$. At least one eigenvalue $\lambda$ of $L$ is real. Let $x \in \mathbb{R}^{3}$ be a corresponding eigenvector. Then $L x=\lambda x$ and so $L^{n} x=\lambda^{n} x$ for all powers $n$. Hence $M x=e^{L} x=e^{\lambda} x$. But then the positive real number $e^{\lambda}$ must be the eigenvalue 1 of $M$; that is $\lambda=0$. Since no other positive real eigenvalue of $M$ exists, the other two eigenvalues of $L$ must be complex conjugate numbers $\mu$ and $\bar{\mu}$. Again, if $y \in \mathbb{C}^{3}$ is an eigenvector of $L$ with respect to $\mu$, then $M y=e^{\mu} y$ implies that $e^{\mu} \in\{-1,-2\}$. But $\bar{y}$ is the conjugate eigenvector of $L$ with respect to $\bar{\mu}$ and so $M \bar{y}=e^{\bar{\mu}} \bar{y}$. It can't be the case, though, that $\left\{e^{\mu}, e^{\bar{\mu}}\right\} \subseteq\{-1,2\}$. Thus we achieved a contradiction. We conclude that $\pm M$ is not a real exponential.

It is interesting to note that the matrix

$$
\tilde{M}=\left(\begin{array}{ccc}
-1 & 0 & 0 \\
0 & -1 & 0 \\
0 & 0 & 1
\end{array}\right)
$$

does have a logarithm in $M_{3}(\mathbb{R})$. In fact, since $\tilde{L}^{2}=-\pi^{2} \tilde{L}$, it is straightforwad to check that

$$
\tilde{L}=\left(\begin{array}{ccc}
0 & -\pi & 0 \\
\pi & 0 & 0 \\
0 & 0 & 0
\end{array}\right)
$$

is a logarithm of $\tilde{M}$. 
An explicit description of those matrices in $M_{n}(\mathbb{R})$ having a real logarithm is given in [2]. It tells us that $M \in M_{n}(\mathbb{R})$ with $\operatorname{det} M>0$ has a logarithm in $M_{n}(\mathbb{R})$ if and only if every Jordan block associated with a negative eigenvalue of $M$ appears an even number of times.

\section{More ON REAL MATRICES}

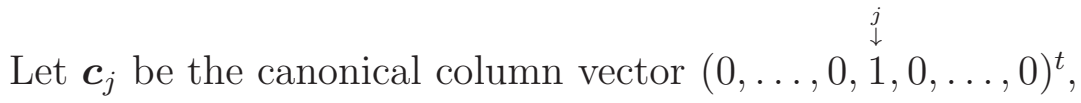

$$
I_{n}=\left(\begin{array}{lll}
1 & & 0 \\
& \ddots & \\
0 & & 1
\end{array}\right)=\left(\boldsymbol{c}_{1}, \ldots, \boldsymbol{c}_{n}\right)
$$

the identity matrix and

$$
\tilde{I}_{n}:=\left(\begin{array}{cccc}
-1 & & & 0 \\
& 1 & & \\
& & \ddots & \\
0 & & & 1
\end{array}\right)=\left(-\boldsymbol{c}_{1}, \boldsymbol{c}_{2}, \ldots, \boldsymbol{c}_{n}\right) .
$$

It is well known that the group $G_{M_{n}(\mathbb{R})}$ of invertible real matrices has exactly two components:

\section{Proposition 3.1.}

$$
\begin{aligned}
& G^{+}=\left\{M \in G_{M_{n}(\mathbb{R})}: \operatorname{det} M>0\right\}=\left\{\prod_{j=1}^{m} e^{M_{j}}: m \in \mathbb{N}^{*}, M_{j} \in M_{n}(\mathbb{R})\right\} \\
& G^{-}=\left\{M \in G_{M_{n}(\mathbb{R})}: \operatorname{det} M<0\right\}=\left\{\tilde{I}_{n} \prod_{j=1}^{m} e^{M_{j}}: m \in \mathbb{N}^{*}, M_{j} \in M_{n}(\mathbb{R})\right\} .
\end{aligned}
$$

Below we shall not only give a simple proof of this fact, but also obtain the result that every matrix in $G^{+}$actually is a product of at most two exponential matrices ${ }^{3}$. We will use a weak version of Schur's reduction theorem for matrices (see for instance [1] for a proof of the general case):

Theorem 3.2. If $M \in M_{n}(\mathbb{R})$ and if $M$ admits $k$ real eigenvalues $\lambda_{1}, \ldots, \lambda_{k}$, $(1 \leq k \leq n)$, then there exists an orthogonal matrix $Q$ such that

$$
Q^{t} M Q=\left(\begin{array}{ccc|cc}
\lambda_{1} & & * & * & * \\
& \ddots & & & \\
0 & & \lambda_{k} & * & * \\
\hline 0 & & 0 & * & * \\
\vdots & & \vdots & & \\
0 & & 0 & * & *
\end{array}\right)
$$

\footnotetext{
3 This is probably known among the workers in the field, but we couldn't trace it in the literature.
} 
Theorem 3.3. Every matrix $M$ in $M_{n}(\mathbb{R})$ with $\operatorname{det} M>0$ is a product of two exponential matrices in $M_{n}(\mathbb{R})$.

Proof. Let $\lambda_{1}, \ldots, \lambda_{k}$ be the negative eigenvalues of $M$ (listed according to their multiplicities). If there are none, Theorem 2.1 (2) implies the existence of a real logarithm. So in this case, the second exponential is $I_{n}$. Thus we may assume $k \geq 1$.

By Theorem 3.2 there is an orthogonal matrix $Q \in M_{n}(\mathbb{R})$ such that

$$
A:=Q^{t} M Q=\left(\begin{array}{ccc|cc}
\lambda_{1} & & * & * & * \\
& \ddots & & & \\
0 & & \lambda_{k} & * & * \\
\hline 0 & & 0 & * & * \\
\vdots & & \vdots & & \\
0 & & 0 & * & *
\end{array}\right) .
$$

Since the characteristic polynomial

$$
p_{M}(\lambda)=\operatorname{det}\left(M-\lambda I_{n}\right)=(-1)^{n} \prod_{j=1}^{n}\left(\lambda-\lambda_{j}\right)
$$

evaluated at $\lambda=0$ equals $\operatorname{det} M=\prod_{j=1}^{n} \lambda_{j}$, and since non-real zeros occur in pairs $(\lambda, \bar{\lambda})$, we conclude that the number of negative eigenvalues of $M$ is even. Let $P$ be the diagonal matrix

$$
P=\left(-c_{1}, \ldots,-\boldsymbol{c}_{k}, \boldsymbol{c}_{k+1}, \ldots \boldsymbol{c}_{n}\right),
$$

where, as we know, $k \in 2 \mathbb{N}$. Then

$$
P A=\left(\begin{array}{ccc|cc}
\left|\lambda_{1}\right| & & * & * & * \\
& \ddots & & & \\
0 & & \left|\lambda_{k}\right| & * & * \\
\hline 0 & & 0 & * & * \\
\vdots & & \vdots & & \\
0 & & 0 & * & *
\end{array}\right),
$$

and the eigenvalues of $P A$ are $\left|\lambda_{1}\right|, \ldots,\left|\lambda_{k}\right|, \lambda_{k+1}, \ldots, \lambda_{n}$. Note that the lower right block of $A$ did not change. Thus $\sigma(P A) \cap]-\infty, 0]=\emptyset$. By Theorem 2.1 (2), $P A=e^{B}$ for some $B \in M_{n}(\mathbb{R})$. Moreover, it is easy see that $P$ has a real logarithm, too, say $P=e^{C}$ (just use the block-matrix structure and the formula

$$
\exp \left(\begin{array}{cc}
0 & -t \\
t & 0
\end{array}\right)=\left(\begin{array}{cc}
\cos t & -\sin t \\
\sin t & \cos t
\end{array}\right)
$$

and put $t=\pi$, respectively $t=0)$. Hence, by noticing that $Q^{t} Q=I_{n}$ and $P P=I_{n}$,

$$
M=Q A Q^{t}=Q(P P A) Q^{t}=Q\left(e^{C} e^{B}\right) Q^{t}=e^{Q C Q^{t}} e^{Q B Q^{t}} .
$$


Corollary 3.4. If $M \in M_{n}(\mathbb{R})$ has a negative determinant, then there are $B_{1}, B_{2} \in M_{n}(\mathbb{R})$ such that $M=\tilde{I}_{n} e^{B_{1}} e^{B_{2}}$.

Proof. Just consider the matrix $\tilde{M}:=\tilde{I}_{n} M$, for which $\operatorname{det} \tilde{M}>0$ and use the previous Theorem.

Proposition 3.1 now immediately follows since $t \mapsto e^{t B_{1}} e^{t B_{2}}$ is a path in $G^{+}$ joining $I_{n}$ with $M$ (and similarily for $G^{-}$). Just note that by Jacobi's trace formula $\operatorname{det} e^{A}=e^{\operatorname{trace} A}>0$ for $A \in M_{n}(\mathbb{R})$.

As a corollary to Theorem 2.1 (or as a special case to Corollary 2.2), we obtain the following result:

Corollary 3.5. A real matrix $M \in M_{n}(\mathbb{R})$ has a logarithm if and only if $M=A^{2}$ for some $A \in M_{n}(\mathbb{R})$.

Proof. If $M=e^{B}$ for some $B \in M_{n}(\mathbb{R})$, then $A:=e^{B / 2}$ has the desired property that $M=A^{2}$. On the other hand, if $M=A^{2}$, then by Theorem 2.1, $A^{2}$ has a logarithm (note that the real-symmetric spectrum $\sigma_{M_{n}(\mathbb{R})}^{*}(A)$ is finite and so it obviously satisfies the assumptions of that Theorem).

As we have seen, if $A \in M_{n}(\mathbb{R})$ with $\operatorname{det} A>0$, then $A=e^{B_{1}} e^{B_{1}}$ where $B_{j} \in M_{n}(\mathbb{R})$, but $A$ does not necessarily belong to $\exp M_{n}(\mathbb{R})$. The following result, though, shows that each $A \in G_{M_{n}(\mathbb{R})}$ comes very close to admit a real logarithm.

Corollary 3.6. Let $A$ be an invertible matrix in $M_{n}(\mathbb{R})$. Then the $(2 n) \times(2 n)$ matrix

$$
M:=\left(\begin{array}{cc}
A & O \\
O & A
\end{array}\right)
$$

has a logarithm in $M_{2 n}(\mathbb{R})$.

Proof. First we note that $\operatorname{det} M=(\operatorname{det} A)^{2} \neq 0$. Hence $M$ is invertible in $M_{2 n}(\mathbb{R})$. Let $H \in M_{n}(\mathbb{C})$ be a complex logarithm of $A$. Put $C:=\operatorname{Re} e^{H / 2}$ and $D:=$ $\operatorname{Im} e^{H / 2}$. Using Corollary 3.5, it suffices to show that

$$
\left(\begin{array}{ll}
A & O \\
O & A
\end{array}\right)=\left(\begin{array}{cc}
C & -D \\
D & C
\end{array}\right)^{2}
$$

Note that

$$
\left(\begin{array}{cc}
C & -D \\
D & C
\end{array}\right)^{2}=\left(\begin{array}{cc}
C^{2}-D^{2} & -C D-D C \\
D C+C D & C^{2}-D^{2}
\end{array}\right)
$$

Using that $C=(1 / 2)\left(e^{H / 2}+e^{\bar{H} / 2}\right)$ and $D=(-i / 2)\left(e^{H / 2}-e^{\bar{H} / 2}\right)$, we see that $C D+D C=0$ and

$$
C^{2}-D^{2}=(C+i D)^{2}=\left(e^{H / 2}\right)^{2}=e^{H}=A .
$$

Acknowledgement. We thank Amol Sasane for providing us with reference $[2]$. 


\section{REFERENCES}

1. R. Bellman Introduction to matrix analysis, Reprint of the second (1970) edition. Classics in Applied Mathematics, 19. Society for Industrial and Applied Mathematics (SIAM), Philadelphia, PA, 1997.

2. W.J. Culver, On the existence and uniqueness of the real logarithm of a matrix, Proc. Amer. Math. Soc. 17 (1966) 1146-1151.

3. S.H. Kulkarni, B.V. Limaye. Real Function algebras, Marcel Dekker, New York, 1992.

4. W. Rudin Functional Analysis, Second Edition, MacGraw-Hill, 1991.

1 Université de Lorraine, DÉpartement de Mathématiques et Institut Élie Cartan de Lorraine, UMR 7502, Ile du Saulcy, F-57045 Metz, France

E-mail address: raymond.mortini@univ-lorraine.fr

2 Fakultät für Angewandte Mathematik, Physik und Allgemeinwissenschaften, TH-Nürnberg, Kesslerplatz 12, D-90489 Nürnberg, Germany

E-mail address: Rudolf.Rupp@th-nuernberg.de 